\title{
Distance Bounding with IEEE 802.15.4a: Attacks and Countermeasures
}

\author{
Marcin Poturalski*, Manuel Flury*, Panos Papadimitratos, Jean-Pierre Hubaux, and Jean-Yves Le Boudec
}

\begin{abstract}
Impulse Radio Ultra-Wideband, in particular the

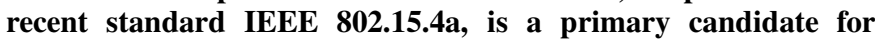
implementing distance bounding protocols, thanks to its ability to perform accurate indoor ranging. Distance bounding protocols allow two wireless devices to securely estimate the distance between themselves, with the guarantee that the estimate is an upper-bound on the actual distance. These protocols serve as building blocks in security-sensitive applications such as tracking, physical access control, or localization.

We investigate the resilience of IEEE 802.15.4a to physicalcommunication-layer attacks that decrease the distance measured by distance bounding protocols, thus violating their security. We consider two attack types: malicious prover (internal) and distance-decreasing relay (external). We show that if the honest devices use energy-detection receivers (popular due to their low cost and complexity), then an adversary can perform highly effective internal and external attacks, decreasing the distance by hundreds of meters. However, by using more sophisticated rake receivers, or by implementing small modifications to IEEE 802.15.4a and employing energy-detection receivers with a simple countermeasure, honest devices can reduce the effectiveness of external distance-decreasing relay attacks to the order of $10 \mathrm{~m}$. The same is true for malicious prover attacks, provided that an additional modification to IEEE 802.15.4a is implemented.
\end{abstract}

Index Terms-Security, ranging, distance bounding, impulse radio, ultra-wideband.

\section{INTRODUCTION}

$\mathbf{O}$ $\mathrm{NE}$ of the distinguishing features of Impulse Radio Ultra-Wideband (IR-UWB) is its capability of high precision indoor ranging, even in dense multi-path environments [1]. This was also one of the main reasons for including an IR-UWB physical layer (PHY) in the IEEE 802.15.4a standard. The ability to measure the distance between two devices with a precision of less than one meter is an enabler for many location-aware applications and services, such as physical access control, tracking of goods and people, or indoor localization. Many of these applications are securitysensitive: They require trustworthy distance measurements, even in the presence of an adversary interfering with the ranging process.

*Equally contributing authors.

Manuscript received July 7, 2010; revised September 30, 2010; accepted December 13, 2010. The associate editor coordinating the review of this paper and approving it for publication was W. Lou.

M. Poturalski, M. Flury, J.-P. Hubaux, and J.-Y. Le Boudec are with the Laboratory for Computer Communications and Applications, EPFL, Switzerland (e-mail: marcin.poturalski@epfl.ch).

P. Papadimitratos is with the School of Electrical Engineering, KTH, Stockholm, Sweden (e-mail: papadim@kth.se).

The work presented in this paper was supported (in part) by the National Competence Center in Research on Mobile Information and Communication Systems (NCCR-MICS), a center supported by the Swiss National Science Foundation under grant number 5005-67322.

Digital Object Identifier 10.1109/TWC.2011.11.101219
Secure range estimation is the domain of distance-bounding (DB) protocols [2]. They allow a verifier to obtain a secure upper-bound on the distance to a prover. $\mathrm{DB}$ protocols are cryptographic in nature, which means that they consider an adversary that manipulates and injects messages on the bit level. However, DB protocols abstract away from the PHY details and are therefore susceptible to PHY attacks, in which an adversary manipulates PHY symbols, rather than bits [3]. Such attacks can bypass any cryptographic mechanisms and allow a malicious prover or an external adversary mounting a relay attack to violate DB security by decreasing the measured distance.

Because of its unique ranging capabilities, IR-UWB is often mentioned as an ideal candidate for a DB PHY. In addition, IR-UWB can incorporate very short symbols, which mitigates PHY attacks [3]. One approach is to design an IR-UWB DB PHY from scratch. An alternative approach, which we investigate in this paper, is to use an existing PHY, such as IEEE 802.15.4a. The latter approach has a number of advantages. First, PHYs are typically designed with performance in mind, and a DB protocol would benefit from the performance optimization provided by such a PHY. Another important benefit is the ease of deployment on devices compatible with an established PHY. However, a thorough evaluation of resilience of the PHY to PHY attacks is crucial: Often, features improving benign-case performance create vulnerabilities.

This is exactly the problem we address in this paper: We adapt the distance-decreasing PHY attacks introduced in [3] (early detection and late commit) to the IEEE 802.15.4a standard and evaluate their effectiveness. We extend our previous work [4] by considering different types of receivers, and malicious prover attacks (internal) in addition to distancedecreasing relay attacks (external). We also examine countermeasures that can mitigate PHY attacks, while minimally degrading the benign-case performance. We make the following new contributions:

- We unveil an anomaly in the convolutional code employed in IEEE 802.15.4a, which can be exploited by an adversary equipped with a rake receiver that attacks energy-detection receivers. It allows the adversary to decrease the distance more substantially than the attacks in [4], or to mount an undetectable attack with a more modest, but still substantial distance-decrease. The anomaly can be patched with a small modification to IEEE 802.15.4a.

- We show that time-hopping used in IEEE 802.15.4a allows a malicious prover to augment the distance-decrease of PHY attacks at the cost of decreasing the probability of attack success. 
A rake-equipped malicious prover can also similarly exploit the combination of BBPM and BPSK employed in IEEE 802.15.4a. The time-hopping vulnerability can be patched with a small modification to IEEE 802.15.4a.

- We observe that by increasing the length of nonces used in a DB protocol, we can improve its performance and preserve the security level. We use this observation to advocate a simple, efficient, and standard-compliant countermeasure to PHY attacks. Employed in an energy-detection receiver, along with the convolutional code patch, this countermeasure effectively limits the distance-decrease of distance-decreasing relay attacks to around $10 \mathrm{~m}$. Employed in an energy-detection receiver or in a rake receiver, along with the convolutional code and time-hopping patches, it limits the effectiveness of malicious prover attacks to around $10 \mathrm{~m}$.

The paper is organized as follows: In Section II we describe the related work. In Section III we discuss our assumptions about DB protocols, and describe a general method to balance performance and security. In Section IV we introduce the IEEE 802.15.4a PHY and the receivers we consider, and explain implementing DB within IEEE 802.15.4a. We show a range of PHY attacks in Section V, and evaluate their performance in Section VI. In Section VII we discuss countermeasures, before concluding in Section VIII. An extended version of this paper is available as a technical report [5].

\section{RELATED WORK}

Distance bounding was first proposed by Brands and Chaum in [2]. The first proposal tolerating errors in the bit-exchange was [6]. A number of other DB protocols are proposed, addressing aspects such as mutual ranging [7], [8], resilience to the terrorist fraud (see Section III) [9], [10], [11], efficiency [12], privacy [13], and formal verification [14], [15].

Physical layer attacks against distance bounding were first introduced in [3]. The effectiveness of these attacks against concrete PHYs is studied in [16] (ISO 14443 RFID and wireless sensor networks) and [4] (IEEE 802.15.4a). Another type of PHY attacks against IR-UWB ranging was introduced in [17]. The attack is based on introducing malicious interference to manipulate the time-of-arrival estimation, thus decreasing the measured distance.

An IR-UWB architecture for implementing DB protocols is proposed in [18]. The maximum distance-decrease an adversary can gain against this PHY is $3-6 \mathrm{~m}$. This is achieved with the short symbol duration of 20ns, which limits the applicability of this PHY in dense multi-path environments for which IEEE 802.15.4a was designed. An ID-based distance bounding protocol is implemented on proprietary IR radios in [19]. Beyond IR-UWB, DB PHYs tailored to narrow-band RFID systems are proposed in [10], [20], [21], and a DB PHY for smartcards (wire-line) is introduced in [22].

\section{Distance Bounding}

Distance bounding (DB) protocols are cryptographic protocols that allow one device, the verifier $V$, to compute an upper-bound on the distance to another device, the prover $P$, in an adversarial setting. Like regular ranging protocols,
DB protocols perform distance estimation based on time-offlight measurements of ranging messages: the challenge(s) sent by $V$ and the response( $s)$ of $P$. Additional messages are employed to guarantee authentication. Three threat scenarios are traditionally considered [23], [9]. In the mafia fraud, the adversary interferes with a DB session between an honest $V$ and an honest $P$, and decreases the measured distance below the actual distance. In the other scenarios, a malicious $P$ convinces $V$ that it is closer than it actually is, working alone (distance fraud), or in collusion with other malicious devices (terrorist fraud).

\section{A. Security Level and Performance}

The challenge and response messages in a DB protocol (Figure 1) are typically nonces (of length $N_{\text {nonce }}$ each), unpredictable to the adversary. At the end of the protocol execution, the verifier learns both the true and the received values of these nonces. The verifier accepts a distance measurement only if the received challenge and response messages contain less than $N_{\text {err }}$ erroneous bits each. The parameters $N_{\text {nonce }}, N_{\text {err }}$ jointly determine 1) the maximum bit error rate (BER) that the protocol tolerates and 2) the security level.

The security level of a DB protocol is defined as the probability that the adversary will succeed in decreasing the measured distance below the actual distance. Let us set aside physical layer attacks, and assume that the DB protocol is cryptographically secure and that the authenticator is too long to be guessable. Then, the adversary is limited to guessing attacks on the nonces: 1) a malicious $P$ guesses the challenge or response and replies early to $V$ 's challenge; 2) an external adversary guesses the response and replies early in place of the honest $P ; 3$ ) an external adversary guesses the challenge, sends it to $P$ to extract the correct response, and sends this response to $V$. The success probability of such guessing attacks is:

$$
P_{\text {guess }}=F_{\mathrm{BIN}}\left(N_{\mathrm{err}} \mid N_{\text {nonce }}, \frac{1}{2}\right)
$$

where $F_{\mathrm{BIN}}(x \mid n, p)$ is the CDF of a binomial distribution with parameters $n$ and $p$.

Inverting the CDF yields the maximum $N_{\text {err }}$ achieving a desired security level $P_{\text {guess }}$ :

$$
N_{\text {err }}=F_{\text {BIN }}^{-1}\left(P_{\text {guess }} \mid N_{\text {nonce }}, \frac{1}{2}\right)
$$

Interestingly, for a fixed security level, this allows a DB protocol to operate at virtually any bit error rate by simply increasing $N_{\text {nonce }}$ and $N_{\text {err }}$. (We will see in Section VII why this is an important property for potential countermeasures). Indeed, we have that:

$$
\begin{aligned}
\lim _{N_{\text {nonce }} \rightarrow \infty} N_{\text {err }} & =\lim _{N_{\text {nonce } \rightarrow \infty}} F_{\text {BIN }}^{-1}\left(P_{\text {guess }} \mid N_{\text {nonce }}, 1 / 2\right)= \\
& =\lim _{N_{\text {nonce }} \rightarrow \infty} F_{\mathcal{N}}^{-1}\left(P_{\text {guess }} \mid N_{\text {nonce }} / 2, N_{\text {nonce }} / 4\right)
\end{aligned}
$$

where $F_{\mathcal{N}}^{-1}\left(x \mid \mu, \sigma^{2}\right)$ is the inverse of the CDF of a normal distribution with mean $\mu$ and variance $\sigma^{2}$ and the second equality follows from the central limit theorem. The protocol succeeds as long as there are no more than $N_{\text {err }}$ errors in a nonce of length $N_{\text {nonce, }}$, resulting in a bit error rate of $\mathrm{BER}^{\max }=N_{\text {err }} / N_{\text {nonce}}$. From (3) it then follows that, as the 

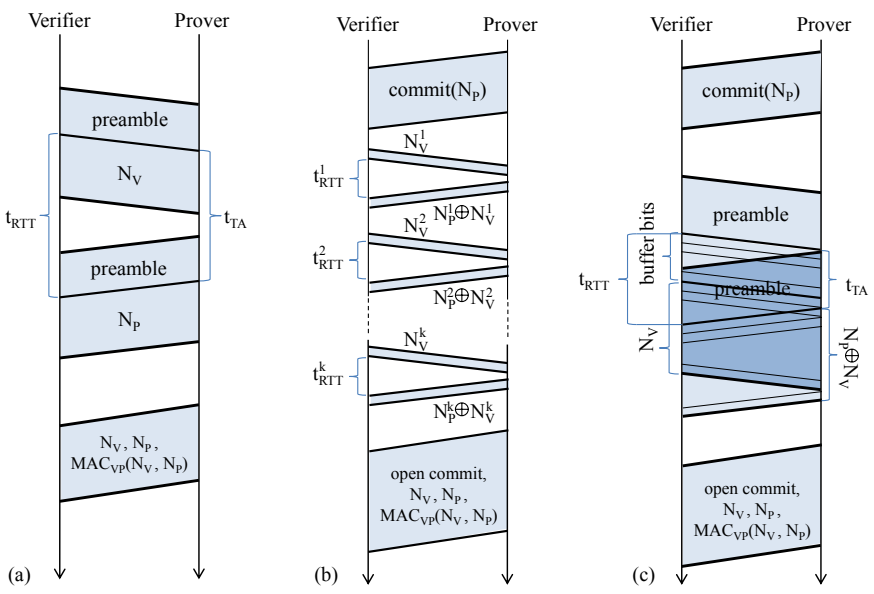

Fig. 1. Examples of distance bounding protocol: (a) is secure only against the mafia fraud, (b) and (c) are secure against the distance fraud and the mafia fraud. (b) achieves distance fraud resilience with the rapidbit-exchange (RBE), (c) replaces RBE with full-duplex transmission. All protocols are simplified for the sake of clarity, in reality both the Verifier, $\mathbf{V}$, and Prover, $\mathbf{P}$, need to be aware that they are engaging in a distance bounding session with each other. $\mathbf{V}$ estimates the distance to $\mathbf{P}$ with the formula $d_{V P}=c\left(t_{\mathrm{RTT}}-t_{\mathrm{TA}}\right) / 2$, where $c$ is the channel propagation speed. $\mathrm{MAC}_{\mathrm{VP}}$ stands for Message Authentication Code with a symmetric key shared between $\mathbf{V}$ and $\mathbf{P}, N_{V}$ and $N_{P}$ are freshly generated nonces, $t_{\mathrm{TA}}$ is a constant turn-around time that $\mathbf{V}$ and $\mathbf{P}$ know, and which is assumed 0 for protocol (b), and $t_{\mathrm{RTT}}$ is the round-trip-time measured by $\mathbf{V}$.

length of the nonce increases, the maximum sustainable bit error rate $\mathrm{BER}^{\mathrm{max}}$ tends to the worst case of $1 / 2$, i.e.,

$$
\begin{aligned}
& \lim _{N_{\text {nonce }} \rightarrow \infty} \mathrm{BER}^{\max }= \\
& \lim _{N_{\text {nonce }} \rightarrow \infty} \frac{N_{\text {nonce }} / 2+\sqrt{N_{\text {nonce }} / 4} \Phi^{-1}\left(P_{\text {guess }}\right)}{N_{\text {nonce }}}=\frac{1}{2}
\end{aligned}
$$

where $\Phi^{-1}(x)$ denotes the inverse CDF of a standard normal distribution.

\section{B. Choosing the Nonce Length}

DB protocols make use of both ranging and communication packets. We have seen in the preceding section that ranging packets carrying nonces can support very high bit error rates and still achieve the desired security level $P_{\text {guess }}$, provided that the coding rate is properly adjusted through the parameters $N_{\text {nonce }}$ and $N_{\text {err }}$. In contrast, communication packets do not necessarily offer the same flexibility because the coding rate can be fixed (as in the case of IEEE 802.15.4a, see Section IV-A3). Consequently, if we define a performance goal in terms of the maximum tolerable packet error rate for communication packets of a given length $\mathrm{PER}_{\text {comm }}$, there is a minimum signal-to-noise ratio (SNR) $\mathrm{SNR}_{\min }$ required to reach this goal. Given $\mathrm{PER}_{\text {comm }}, \mathrm{SNR}_{\min }$ can be established analytically or with simulations.

We can define a similar performance goal for ranging packets by fixing their maximum tolerable packet error rate $\mathrm{PER}_{\mathrm{db}}$. Now, if ranging and communication ought to have the same operating range, ranging packets should achieve $P_{\mathrm{db}}$ at $\mathrm{SNR}_{\min }$. To guarantee this, we can first establish (again analytically or with simulations) the bit error rate $\mathrm{BER}_{\mathrm{db}}$ that ranging messages experience at $\mathrm{SNR}_{\min }$. A ranging packet of length $N_{\text {nonce }}$ subject to $\mathrm{BER}_{\mathrm{db}}$ is considered to be in error if

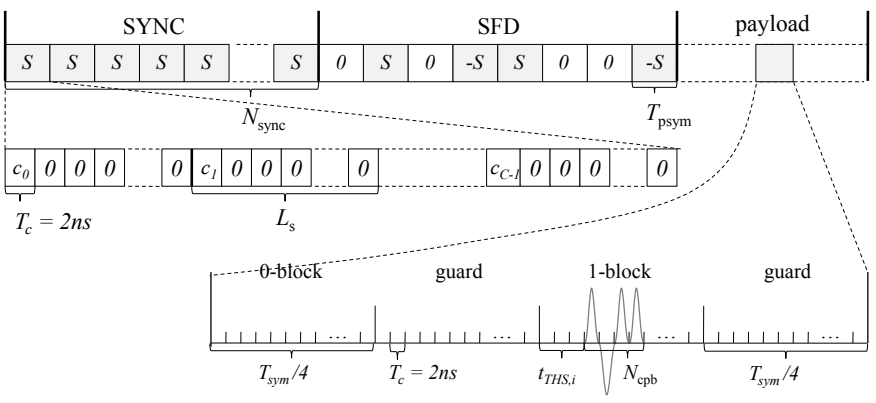

Fig. 2. IEEE 802.15.4a packet structure: preamble and payload.

it contains more than $N_{\text {err }}$ errors. We can thus derive $N_{\text {nonce }}$ and $N_{\text {err }}$ by solving the system of equations formed by (2), that ensures the desired security level, and

$$
N_{\text {err }}=F_{\text {BIN }}^{-1}\left(1-\operatorname{PER}_{\mathrm{db}} \mid N_{\text {nonce }}, \mathrm{BER}_{\mathrm{db}}\right)
$$

that ensures the required $\mathrm{PER}_{\mathrm{db}}$.

\section{IR-UWB System Model AND Assumptions}

\section{A. IEEE 802.15.4a PHY}

IEEE 802.15.4 is a standard for low-rate wireless personal area networks (WPAN). The IEEE 802.15.4a amendment [24] defines an IR-UWB PHY that allows for low-rate communication and high precision ranging. Because of the ultrawideband nature of this PHY, the transmitting power is significantly limited by regulation. This results in a relatively low communication range $(20-30 \mathrm{~m})$. We focus on one of the mandatory modes of the standard, LPRF (low pulse repetition frequency). However, our results are easily transferable to other modes (which use different parameter values). All parameter values are publicly known.

An IEEE 802.15.4a packet (Figure 2) is composed of a preamble followed by a payload part.

1) Preamble: The preamble consists of a SYNC part and the start frame delimiter (SFD). The SYNC part is composed of $N_{\text {sync }}=64$ identical preamble symbols of duration $T_{\mathrm{psym}}=$ 3968 ns. The SFD is composed of a particular sequence of $N_{\text {sfd }}=8$ preamble symbols. Each preamble symbol is formed by $C$ code symbols that consist of $L_{\mathrm{s}}$ chips of duration $T_{\mathrm{c}}=$ 2 ns. Pulses are sent in the first chip of every code symbol and modulated according to a ternary preamble code of length $C$. The received signal during reception of a preamble and after filtering with a bandpass filter of bandwidth $B$ is given by:

$$
r_{\mathrm{pre}}(t)=\sum_{i=1}^{N_{\mathrm{synn}}+N_{\mathrm{sfd}}} s_{i} \sum_{j=1}^{C} c_{j} \cdot h\left(t-j L_{\mathrm{s}} T_{\mathrm{c}}-i T_{\mathrm{psym}}-\nu_{0}\right)+w(t)
$$

where $h(t)$ is the unknown channel response (including the transmitted waveform, the response of the multi-path channel and the bandpass filter) assumed invariant for the duration of one packet, $w(t)$ is a zero-mean AWGN process with power spectral density $N_{0} / 2, c_{j} \in\{-1,0,+1\}$ are the elements of the preamble code and $\nu_{0}$ is the propagation delay. Each preamble symbol is modulated by the sequence $s_{i}=[1, \ldots, 1,0,1,0,-1,1,0,0,-1]$ whose last eight elements denote the SFD. 
2) Payload: The payload is modulated using a combination of Binary Pulse Position Modulation (BPPM) and Binary Phase-Shift Keying (BPSK). In addition, time-hopping is used to allow for multiple-access and every symbol is signalled through the transmission of a burst of $N_{\mathrm{cpb}}$ pulses. The signal received during the $i$-th payload symbol is:

$r_{i}(t)=w(t)+$

$\left(2 a_{i}-1\right) \sum_{j=1}^{N_{\mathrm{cpb}}} b_{i j} \cdot h\left(t-i T_{\mathrm{sym}}-d_{i} T_{\mathrm{sym}} / 2-t_{\mathrm{THS}, i}-j T_{\mathrm{c}}-\nu_{0}\right)$

where $a_{i}$ is the polarity bit (BPSK), $d_{i}$ the position bit (BPPM), $T_{\text {sym }}=1024 n s$ is the symbol duration, $t_{\mathrm{THS}, i} \in$ $\left[0, t_{\mathrm{THS}}^{\max }\right]$, where $t_{\mathrm{THS}}^{\mathrm{max}}=T_{\mathrm{sym}} / 4-N_{\mathrm{cpb}} \cdot T_{\mathrm{c}}$, defines the pseudorandom time-hopping offset, and the scrambling sequence $b_{i j}$ defines the polarity of the $j$-th pulse of the $i$-th burst. Both the time-hopping and the scrambling sequences are derived from a fixed and publicly known linear feedback shift register that is initialized to a publicly known state at the beginning of every packet. Both sequences are thus the same for every packet.

3) Channel Coding: A systematic rate $1 / 2$ convolutional code with generator polynomials $g_{1}=(0,1,0)$ and $g_{2}=$ $(1,0,1)$ is used. Denote the bits to be transmitted by $x_{i}$. Then the position bit is $d_{i}=x_{i}$ and the polarity bit is $a_{i}=x_{i-1} \oplus x_{i+1}$, where $\oplus$ denotes modulo two addition. With this construction, an energy-detection receiver, which cannot recover the polarity bit, can still decode the transmitted bit sequence $x_{i}$; whereas a coherent receiver, which can recover both bits, can apply convolutional decoding to improve performance. IEEE 802.15.4a also applies a systematic $(55,63)$ Reed-Solomon (RS) code before modulation.

\section{B. Wireless Transceivers}

Honest devices are equipped with an IEEE 802.15.4a compliant receiver and transmitter. The choice of transmitter is of little consequence to our investigation, any standardcompliant transmitter is acceptable, e.g., [25], [26]. We consider two types of receivers: a low-complexity and low-cost non-coherent energy-detection receiver, and a sophisticated coherent rake receiver.

Adversarial devices are equipped with transmitters similar to the honest devices, but able to send non-standard-compliant pulse sequences and to ignore regulatory transmission power limits. The adversary may further equip his devices with high gain antennas, thus allowing him to increase the SNR observed by both adversarial and honest devices. Such an increase in SNR can also be achieved by the adversary moving his devices closer to the honest devices. The receivers used by the adversary are modified versions of the receivers presented next (see Section V).

1) Energy-Detection Receiver: The energy-detection receiver (we also use the term energy detector) squares and integrates the received signal $r(t)$. The integrator outputs a discrete time sample every $T=T_{\mathrm{c}}=2 \mathrm{~ns}$. Such a sampling rate is high enough to allow for precise ranging.

The receiver employs a traditional synchronization algorithm based on a correlation with the known preamble sequence. After a coarse synchronization, usually achieved on the strongest multi-path component, the receiver undertakes a verification phase. If successful, fine synchronization is performed using a back-search algorithm, to obtain a better estimate of the beginning of the signal. The receiver then performs a period of channel estimation where it estimates the energy-delay profile of the channel by averaging a number of preamble symbols. At the same time it also begins to look for the SFD.

To demodulate the $i$-th BPPM bit $d_{i}$ of the payload, the receiver uses the optimum decision rule from [27], [28], comparing the (weighted) energies in the first and second half of the symbol:

$$
\sum_{m=0}^{M-1} y_{m, i} \cdot p_{m} \underset{d_{i}=1}{d_{i}=0} \sum_{m=0}^{M-1} y_{m+\frac{T_{f}}{2 T_{\mathrm{c}}}, i} \cdot p_{m}
$$

where $y_{m, i}$ denotes the $m$-th discrete sample of the $i$-th symbol. The weighting coefficients $p_{m}$ are derived from the energy-delay profile of the channel. The number of samples to combine is $M=t_{\mathrm{det}} / T$, where $t_{\mathrm{det}}$ defines the detection time: the length of the received signal (per half-symbol) that the receiver uses to demodulate the bits; $t_{\mathrm{det}}$ is chosen to be large enough to account for the channel delay spread. In our simulations we use the non-line-of-sight residential channel model from [29] and set $t_{\mathrm{det}}=60 \mathrm{~ns}$ accordingly.

2) Rake Receiver: The receiver with optimal performance (in a benign setting), but also with the highest complexity, is an all-rake receiver using maximum ratio combining (MRC) [30]. The convolutional code is decoded with the optimal symbol-wise branch metric for BPPM/BPSK given in [31]. For this paper, the crucial difference between an energy-detection receiver and a rake receiver is that the latter can recover the polarity bits $a_{i}$ during payload demodulation. This diminishes the effectiveness of payload PHY attacks against rake receivers (Section V-C), but also opens a new space for payload attacks if a rake receiver is used against energy-detection receivers. In our analysis of the rake receiver, we therefore focus on the payload, assuming perfect synchronization and channel estimation.

Similar to the energy-detection receiver, an important parameter for our analysis is the detection time $t_{\mathrm{det}}$, denoting the portion of the received signal that the rake receiver uses to demodulate a bit. We chose $t_{\text {det }}$ large enough to account for the channel delay spread.

\section{Distance Bounding with IEEE 802.15.4a}

DB protocols that are secure only against the mafia fraud, such as the protocol in Figure 1(a), are directly compatible with IEEE 802.15.4a. This is because they employ a standard packet format for ranging messages [7], [14]. However, protocols that are also secure against the distance fraud or the terrorist fraud are typically not directly compatible with IEEE 802.15.4a. Such protocols need to prevent a malicious prover from decreasing the measured distance by responding prematurely. This is traditionally achieved with a rapid bit exchange (RBE), in which $V$ sends single bit challenges, to which the prover should respond instantly (Figure 1(b)). An IEEE 802.15.4a implementation of the RBE - which requires prefixing every bit with a lengthy preamble - is not only 
inefficient, but also opens a space for packet-level attacks [3]. Nevertheless, recent proposals [13] show that with full duplex transmission, regular packet formats can be used in place of the rapid-bit-exchange. Applying this principle in IEEE 802.15.4a requires adding a number of "buffer" bits at the beginning of the challenge's payload, of total duration equal to the duration of the preamble (Figure 1(c)). This is necessary because the prover cannot start sending the response preamble before it synchronizes and detects the SFD of the challenge preamble, whereas security requires that every response symbol is transmitted instantly after the corresponding challenge symbol.

We define $t_{\text {res }}$ as the time interval at the prover between the start of the reception of a challenge bit and the start of the transmission of the corresponding response bit. To make the response as "instant" as possible with IEEE 802.15.4a, we assume that $t_{\mathrm{res}}=T_{\mathrm{sym}} / 2+t_{\mathrm{THS}}^{\mathrm{max}}+t_{\mathrm{det}}$. This is the smallest $t_{\mathrm{res}}$ sufficient to demodulate symbols with maximal time-hopping offsets.

We assume that the DB protocol has access to the received bit sequence before it is decoded with the error correcting codes (Reed-Solomon, convolutional). In a receiver implementation, this assumption can easily be met as these bits have to be received from the channel in any case. If this assumption is violated, the success probability of guessing attacks increases, because coding can mask some of the erroneously guessed bits.

\section{Distance-Decreasing AtTacks}

Physical layer (PHY) attacks were first introduced as a potential attack vector against distance bounding (DB) in [3]. These attacks rely on two primitives: 1) In early detection $(E D)$, an adversarial receiver (ARX) detects a PHY symbol (e.g., payload symbol) of duration $t_{\text {sym }}$ based only on the beginning part of this symbol of duration $t_{\mathrm{ED}}<t_{\mathrm{sym}}$, where $t_{\mathrm{ED}}$ is the $E D$ delay. This leads to a detection which is less reliable, but also faster than that of a normal receiver (which takes $t_{\text {res }}>t_{\mathrm{ED}}$ of the PHY symbol into account for detection). 2 ) In late commit $(L C)$, only the $\left(t_{\mathrm{sym}}-t_{\mathrm{LC}}\right)$-long end-part of the PHY symbol is modulated based on the intended value of the symbol (e.g., whether it encodes a 0 bit or a 1 bit), and the beginning part is modulated independently of this value. This allows an adversarial transmitter (ATX) to delay the decision about which symbol it transmits by $t_{\mathrm{LC}}$, where $t_{\mathrm{LC}}$ is the LC delay. The PHY symbols generated with LC typically differ from regular symbols, but, if appropriately chosen, they can be demodulated by an honest receiver, albeit with some performance loss.

The first type of PHY attack we consider is an internal attack mounted by a malicious prover (consisting of an ARX and an ATX), which can be used in a distance/terrorist fraud. In this attack, a malicious prover uses ED, LC or their combination to respond prematurely to the verifier's $V$ challenge. This decreases the propagation time measured by $V$ by an offset $t_{\text {gain }}$ that we call the time-gain. The time-gain is equal to $\left(t_{\mathrm{C}}+t_{\mathrm{res}}-t_{\mathrm{ED}}\right) / 2$ for an ED-only attack, $\left(t_{\mathrm{LC}}+t_{\text {res }}-t_{\mathrm{D}}\right) / 2$ for an LC-only attack, and $\left(t_{\mathrm{LC}}+t_{\mathrm{res}}-t_{\mathrm{ED}}\right) / 2$ for an $\mathrm{ED}+\mathrm{LC}$ attack, where $t_{\mathrm{D}}$ and $t_{\mathrm{C}}$ are, respectively, the detection delay

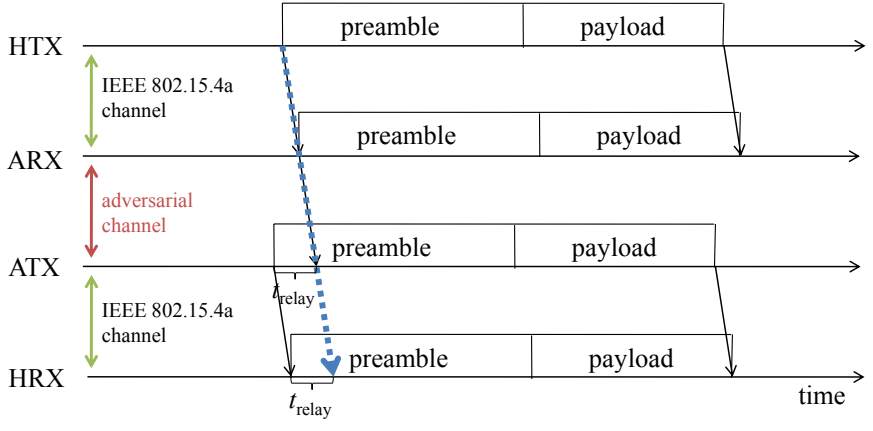

Fig. 3. Overview of the distance-decreasing relay attack. ARX and ATX are assumed to be located on a line between HRX and HTX. The thick dotted arrow indicates time-of-arrival corresponding to the actual distance between HTX and HRX.

and commit delay when the attacker chooses not to perform ED and LC. (Note: it is possible that $t_{\mathrm{D}}<t_{\text {res }}$ and $t_{\mathrm{C}}>0$ if time-hopping is involved.) The time-gain translates into a distance-decrease of $c \cdot t_{\text {gain }}$, where $c$ is the speed of light.

The second type of attack we consider is a distancedecreasing relay attack between two honest devices. This attack is mounted by an external adversary using a combination of ED and LC and it can be classified as a mafia fraud. The general setup for the relay attack is shown in Figure 3 . The adversary should mount the distance-decreasing relay attack on all ranging messages (challenge, response); other messages, being not time-critical, can be relayed in an arbitrary fashion. Without loss of generality, we focus on the exchange of a single ranging message. In this case one of the honest devices acts as a transmitter (HTX) and the other one as a receiver (HRX), whereas one adversarial device acts as an early detection receiver (ARX), and another as a late commit transmitter (ATX). The channel from HTX to ARX, and from ATX to HRX is the IEEE 802.15.4a channel. ARX and ATX communicate using a dedicated, out-of-band adversarial channel. The propagation speed of both channels is $c$, the speed of light.

In a distance-decreasing relay attack the adversary relays messages between HTX and HRX in such a way that to HRX they seem "shifted back in time" by a positive offset $t_{\text {relay }}=t_{\mathrm{LC}}-t_{\mathrm{ED}}$ that we call the relay time-gain (Figure 3 ). The distance measured by $V$ is then reduced by the relay distance-decrease $c \cdot t_{\text {relay }}$. (Assuming that ARX and ATX are located on a line between HTX and HRX. In other configurations the distance decrease will be smaller. Note, however, that the choice of the configuration rests with the adversary.) In the relay attack, ATX needs to begin the transmission of the preamble at time $t_{0}-t_{\text {relay }}$, before ARX begins receiving the preamble from HTX a time $t_{0}$. However, the adversary learns $t_{0}$ only after synchronizing to the preamble of HTX. (Guessing $t_{0}$ is not practical at the nanosecond precision required.) To escape this vicious circle, the adversary needs to mount some form of ED and LC on the preamble, in addition to $\mathrm{ED}$ and $\mathrm{LC}$ on the payload. In contrast, the preamble attacks are not necessary in the case of malicious prover attacks.

Note that the distance-decrease that the adversary might wish to obtain is not limited by the low communication range of IEEE 802.15.4a. Indeed, a malicious prover can increase 


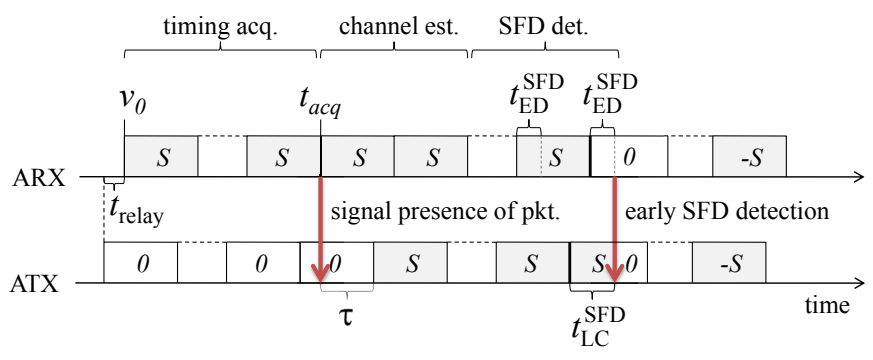

Fig. 4. Distance-decreasing relay attack on the preamble.

his communication range by using a high gain antenna and transmitting with non-regulatory power to reach a remote prover. Further, in a relay attack, the adversary can "connect" remote HRX and HTX by placing ARX close to HTX and ATX close to HRX, and using a long-range ATX-ARX link to which the range limitations of IEEE 802.15.4a do not apply.

We consider three scenarios, in which the adversary uses different types of receivers against different types of receivers used by the honest devices: Energy Detector against Energy Detector, Rake against Energy Detector, and Rake against Rake. For each scenario we first analyze the delay of the ED and LC primitives. Then, we elaborate on the use of these primitives for malicious prover attacks and relay attacks. Table I summarizes the upper-bounds on the time-gain and distance-decrease of various variants of PHY attacks. Note that the preamble attack is only presented in Section V-A1, as this attack is applicable and sufficient in the two other scenarios (it can achieve the relay time-gains of the magnitude required for the payload attack).

\section{A. Energy Detector against Energy Detector}

1) Attack on the Preamble: The attack, which is part of the distance-decreasing relay attack, is depicted in Figure 4; for clarity of presentation, we assume the distance between ARX and ATX to be 0. ARX performs packet detection, timing acquisition, and channel estimation in the same fashion as an honest receiver. ARX then signals the fact that it has acquired timing to ATX. Deviating from honest receivers, ARX performs early SFD detection: It chooses an early SFD detection delay $t_{\mathrm{ED}}^{\mathrm{SFD}}$ and tries to detect the presence of the SFD by deliberately considering only the first $t_{\mathrm{ED}}^{\mathrm{SFD}} \mathrm{ns}$ of every received preamble symbol. As the SFD starts with a 0 modulated preamble symbol, as opposed to a 1 modulated symbol used during the SYNC part, early SFD detection boils down to on-off keying (OOK) demodulation.

At the other end of the relay, ATX chooses a late SFD commit delay $t_{\mathrm{LC}}^{\mathrm{SFD}}$ and remains silent until ARX signals that timing acquisition has been successful. Then, after an appropriately chosen (we explain how shortly) delay $\tau<T_{\text {psym }}$, ATX begins transmitting a sequence of preamble symbols $S$. This is repeated until ARX signals that the SFD was detected. Immediately afterwards, ATX switches to the transmission of a standard compliant SFD, beginning from $t_{\mathrm{LC}}^{\mathrm{SFD}}$ into the $\mathrm{SFD}$. This concludes the distance-decreasing attack on the preamble.

In contrast to a standard-compliant preamble, the SYNC part of the preamble generated by ATX begins with a number of 0 modulated preamble symbols; The beginning of the SFD

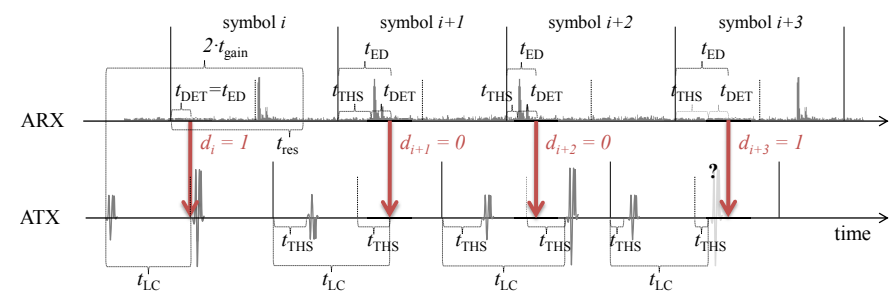

Fig. 5. Example of a malicious prover ED+LC attack on the payload. The first two symbols use equal time-hopping offsets for corresponding symbols, hence their time-gain is identical. The maximal time-gain for the 3rd symbol is larger than the packet-wide time-gain chosen of the adversary. The timegain for the 4th symbol is smaller than the packet-wide time-gain, and the adversary is forced to guess.

corresponds to a 1 modulated preamble symbol for a duration of $t_{\mathrm{LC}}^{\mathrm{SF}}$, instead of having no signal contribution. The relay time-gain achieved by this attack is $t_{\text {relay }}=t_{\mathrm{LC}}^{\mathrm{SFD}}-t_{\mathrm{ED}}^{\mathrm{SFD}}$. This determines the choice of $\tau$, as $T_{\text {psym }}-\tau=t_{\text {relay }} \bmod T_{\text {psym }}$.

2) Attack on the Payload: Payload attacks are performed on a symbol basis. As we are considering energy-detection receivers, which are blind to the signal polarity, only the position bit $d_{i}$ is relevant.

a) ED: ARX performs ED by deciding on the value of $d_{i}^{\mathrm{RX}}$ after an early detection delay $t_{\mathrm{ED}}\left(d_{i}^{\mathrm{RX}}\right)=t_{\mathrm{THS}, i}^{\mathrm{RX}}+t_{\mathrm{det}}^{\mathrm{A}}<$ $T_{\mathrm{sym}} / 2$, where $t_{\mathrm{det}}^{\mathrm{A}}$ denotes the detection time of ARX and $t_{\mathrm{THS}}^{\mathrm{RX}}$ is the time-hopping offset sequence of the received message. This implies that ARX replaces BPPM demodulation with on-off keying (OOK) demodulation. The time $t_{\mathrm{det}}^{\mathrm{A}}$ can be made arbitrarily short, it determines the attack's performance. If ARX chooses not to perform ED, the detection delay is $t_{\mathrm{D}}\left(d_{i}^{\mathrm{RX}}\right)=T_{\mathrm{sym}} / 2+t_{\mathrm{THS}, i}^{\mathrm{RX}}+t_{\mathrm{det}}$.

b) LC: In the LC attack, ATX always transmits a burst of pulses with energy $E_{0}$ (shifted by the appropriate timehopping offset). In the second half of the symbol, ATX acts according to the value of $d_{j}^{\mathrm{TX}}$ : If $d_{j}^{\mathrm{TX}}=0$, ATX transmits nothing in the second part of the symbol; if $d_{j}^{\text {TX }}=1$, ATX transmits a burst of pulses with energy $E_{1}>E_{0}$. This attack exploits the fact that HRX performs a simple energy comparison to demodulate. The late commit delay for $d_{j}^{\mathrm{TX}}$ is $t_{\mathrm{LC}}\left(d_{j}^{\mathrm{TX}}\right)=T_{\mathrm{sym}} / 2+t_{\mathrm{THS}, j}^{\mathrm{TX}}+t_{\mathrm{PLC}}$, where $t_{\mathrm{THS}}^{\mathrm{TX}}$ is the time-hopping offset sequence of the transmitted message, and $t_{\mathrm{PLC}}<t_{\mathrm{det}}$ is the pulse LC delay, by which the transmission of the pulse can be additionally delayed, similar to the LC attacks discussed in [3], [16]. Throughout most of the paper, notably Section VI, we assume $t_{\mathrm{PLC}}=0$. If ATX chooses to send standard-compliant symbols, it can still delay committing to the transmitted symbol by $t_{\mathrm{C}}\left(d_{j}^{\mathrm{TX}}\right)=t_{\mathrm{THS}, j}^{\mathrm{TX}}$.

c) Malicious Prover: The time-gain of the ED-only malicious prover attack for corresponding challenge and response symbols $i$ and $j$ is $t_{\text {gain }}(i, j)=\left(t_{\mathrm{C}}\left(d_{j}^{\mathrm{TX}}\right)+t_{\mathrm{res}}-t_{\mathrm{ED}}\left(d_{i}^{\mathrm{RX}}\right)\right) / 2=$ $\left(T_{\mathrm{sym}} / 2+t_{\mathrm{THS}}^{\mathrm{max}}+t_{\mathrm{det}}-t_{\mathrm{det}}^{\mathrm{A}}\right) / 2+\left(t_{\mathrm{THS}, j}^{\mathrm{TX}}-t_{\mathrm{THS}, i}^{\mathrm{RX}}\right) / 2=C+$ $\left(t_{\mathrm{THS}, j}^{\mathrm{TX}}-t_{\mathrm{THS}, i}^{\mathrm{RX}}\right) / 2$, where $C$ is a constant not dependent on $i$ and $j$. The time-gain of other malicious prover attacks can also be expressed as $C+\left(t_{\mathrm{THS}, j}^{\mathrm{TX}}-t_{\mathrm{THS}, i}^{\mathrm{RX}}\right) / 2$. The latter term varies from $-t_{\mathrm{THS}}^{\max } / 2$ to $t_{\mathrm{THS}}^{\max } / 2$, because $t_{\mathrm{THS}}^{\mathrm{TX}} \neq t_{\mathrm{THS}}^{\mathrm{RX}}$ if different channels are used for for RX and TX, but also because $i \neq j$ due to the "buffer" bits (Figure 1(c)). However, the structure of the attack demands that the adversary chooses a constant time-gain $t_{\text {gain }}$ for all symbols. This leaves the adversary 
with a strategic decision: The adversary can set the time-gain conservatively, to make sure there is enough time to perform $\mathrm{ED}$ and/or LC on every symbol (i.e., choose $t_{\text {gain }} \leq t_{\text {gain }}(i, j)$ for all corresponding $i, j$ index pairs). Alternatively, the adversary can set the time-gain more aggressively, which will force him to guess the bits with unfavorable time-hopping offsets (i.e., $i, j$ pairs for which $\left.t_{\text {gain }}(i, j)<t_{\text {gain }}\right)$. This is illustrated in Figure 5. In this way, the adversary can trade-off a larger time-gain (up to $2 \cdot t_{\mathrm{THS}}^{\max } / 2$ ) for a lower attack success probability. Figure 6 shows this trade-off for one particular case $\left(N_{\text {nonce }}=42, N_{\text {err }}=2\right.$, mandatory LPRF mode).

d) Relay: For the relay attack, the time-gain is $t_{\text {relay }}(i, j)=t_{\mathrm{LC}}\left(d_{j}^{\mathrm{TX}}\right)-t_{\mathrm{ED}}\left(d_{i}^{\mathrm{RX}}\right)=T_{\mathrm{sym}} / 2+t_{\mathrm{PLC}}+$ $t_{\mathrm{THS}, j}^{\mathrm{TX}}-t_{\mathrm{THS}, i}^{\mathrm{RX}}-t_{\mathrm{det}}^{\mathrm{A}}$. However, in the case of the relay attack $t_{\mathrm{THS}}^{\mathrm{RX}}=t_{\mathrm{THS}}^{\mathrm{TX}}$ and $i=j$. Hence, the time gain is $t_{\text {relay }}=T_{\text {sym }} / 2+t_{\mathrm{PLC}}-t_{\mathrm{det}}^{\mathrm{A}}$ for every symbol. This is also the upper-bound on the overall time-gain of the relay attack, as the time-gains achievable for the preamble are larger.

\section{B. Rake against Energy Detector}

1) $E D$ and $L C$ : If honest devices use energy detectors, using a rake receiver allows the adversary to perform an $\mathrm{ED}$ attack with negative delay $t_{\mathrm{ED}}$ by extracting $d_{i}^{\mathrm{RX}}$ from the (i-1)-th symbol. This attack exploits the structure of the convolutional code: The (i-1)-th payload symbol carries the position bit $d_{i-1}^{\mathrm{RX}}$ and the polarity bit $a_{i-1}^{\mathrm{RX}}=d_{i-2}^{\mathrm{RX}} \oplus d_{i}^{\mathrm{RX}}$. With a rake receiver, ARX can decode both bits, and obtain $d_{i}^{\mathrm{RX}}$ by computing $a_{i-1}^{\mathrm{RX}} \oplus d_{i-2}^{\mathrm{RX}}$. This is all that is necessary to transmit the corresponding $j$-th symbol: The adversary can compute $d_{j}^{\mathrm{TX}}$ from $d_{i}^{\mathrm{RX}}$, and $a_{j}^{\mathrm{TX}}$ can be set arbitrarily, as polarity bits cannot be demodulated by an energy-detection receiver. The delay of the rake ED attack extracting $d_{i}$ from the (i-1)-th symbol is $t_{\mathrm{ED}}\left(d_{i}^{\mathrm{RX}}\right)=-\left(1-d_{i-1}^{\mathrm{RX}}\right) \cdot T_{\mathrm{sym}} / 2-T_{\mathrm{sym}} / 2+t_{\mathrm{THS}, i-1}^{\mathrm{RX}}+t_{\mathrm{det}}^{\mathrm{A}}$. HRX is an energy-detector as in Section V-A2, hence the same LC attack applies.

2) Malicious Prover and Relay: With the rake ED attack, the malicious prover attacks, but also the relay attack (as $i=$ $j-1$ in this case) are subject to per-symbol variability of the time-gain due to time-hopping offsets. An additional timegain variability is due to BPPM, i.e., the term " $-\left(1-d_{i-1}^{\mathrm{RX}}\right)$. $T_{\text {sym }} / 2$ " of the ED delay. As in Section V-A2, this presents the adversary with a trade-off between the distance-decrease, and the probability of a successful attack. For example, the additional time-gain of the relay attack is at most $T_{\mathrm{sym}} / 2+2$. $t_{\mathrm{THS}}^{\max }$, and Figure 6 shows the trade-off for a particular set of parameters. See Table I for the malicious prover attack.

Furthermore, a negative ED delay allows for an ED-only distance-decreasing relay attack to be mounted. Although it has a lower time-gain than an ED+LC relay attack, the EDonly attack circumvents any countermeasures that prevent LC attacks, e.g., the countermeasure advocated in Section VII.

\section{Rake against Rake}

To mitigate the effects of the rake ED attack, an honest rake receiver must demodulate and check the correctness of both positions bits $d_{i}$ and polarity bits $a_{i}$ (without applying convolutional decoding, see assumption in Section IV-C). With this precaution in place, ATX cannot transmit symbol $j$ (with or without LC) without knowing $a_{j}^{\mathrm{TX}}$.

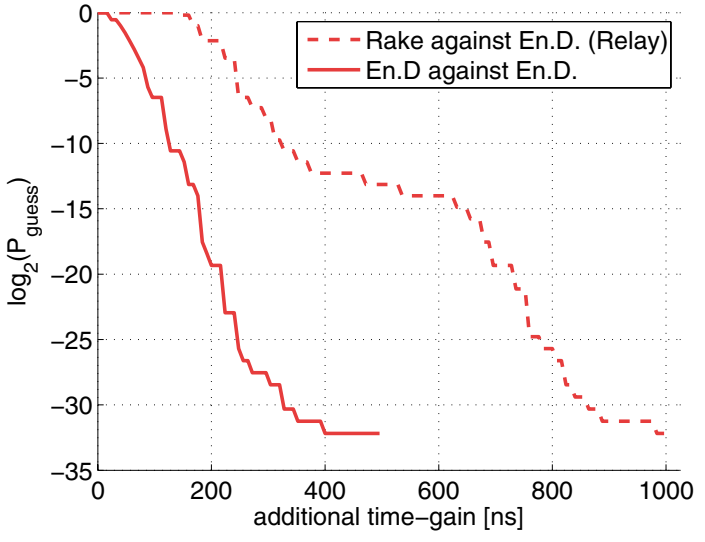

Fig. 6. Example trade-off between guessing probability and additional timegain achievable with 1) Malicious prover attacks in the scenario "Energy Detector against Energy Detector" 2) Distance-decreasing relay attack in the scenario "Rake against Energy Detector". The guessing probability $P_{\text {guess }}\left(t_{\text {gain }}^{+}\right)=F_{\text {BIN }}\left(N_{\text {err }} \mid B\left(N_{\text {nonce }}, t_{\text {gain }}^{+}\right), \frac{1}{2}\right)$, where $B\left(N_{\text {nonce }}, t_{\text {gain }}^{+}\right)$is the number of bits out of $N_{\text {nonce }}$ that the adversary must guess to obtain an additional time-gain $t_{\text {gain }}^{+}$. The timing parameter values (notably the time-hopping sequence) correspond to the mandatory LPRF mode of IEEE 802.15.4a, and $N_{\text {nonce }}=42, N_{\text {err }}=2$ (corresponds to security level $2^{-32}$ )

1) $E D$ and $L C$ : The ED delay of demodulating the polarity bit $a_{i}^{\mathrm{RX}}$ is $t_{\mathrm{ED}}\left(a_{i}^{\mathrm{RX}}\right)=d_{i}^{\mathrm{RX}} \cdot T_{\mathrm{sym}} / 2+t_{\mathrm{THS}, i}^{\mathrm{RX}}+t_{\mathrm{det}}^{\mathrm{A}}$. The LC delay of committing to $a_{j}^{\mathrm{TX}}$ is $t_{\mathrm{LC}}\left(a_{j}^{\mathrm{TX}}\right)=t_{\mathrm{THS}, j}^{\mathrm{TX}}+d_{j}^{\mathrm{TX}} \cdot T_{\mathrm{sym}} / 2+t_{\mathrm{PLC}}$. Note that both delays depend on the value of the position bit $d$. The delays for ED of $d_{i}^{\mathrm{RX}}$ and LC of $d_{j}^{\mathrm{TX}}$ are as in Section V-B.

2) Malicious Prover and Relay: The time-gain is computed as the minimum of the time-gain for the position bits $d$ and the time-gain for the polarity bits $a$. In the relay attack, $i=j$, $d^{\mathrm{RX}}=d^{\mathrm{TX}}, a^{\mathrm{RX}}=a^{\mathrm{TX}}$, and $t_{\mathrm{THS}}^{\mathrm{RX}}=t_{\mathrm{THS}}^{\mathrm{TX}}$, hence the relay time-gain is $t_{\text {relay }}=t_{\mathrm{PLC}}-t_{\mathrm{det}}^{\mathrm{A}}<t_{\mathrm{det}}$, which translates to at most $10 \mathrm{~m}$ assuming $t_{\mathrm{det}}=32 \mathrm{~ns}$ (see Section VI-B). This is an order of magnitude lower than the attacks presented so far. The time-gain of malicious prover attacks, without guessing, is of the same order of magnitude, see Table I. However, a malicious prover (but not a relaying adversary) can increase the distance-decrease by as much as $T_{\mathrm{sym}} / 2+t_{\mathrm{THS}}^{\mathrm{max}}$, by lowering the probability of success.

Furthermore, in the case of the relay attack, if only one of the honest devices uses an energy-detection receiver, notably without any countermeasures deployed, (and even though the other one uses a rake receiver) the adversary can achieve a significant time-gain. Even assuming that the distancedecrease against the rake receiver is negligible, the overall distance-decrease of the relay attack is $c \cdot t_{\text {relay }}^{\text {En.D. }} / 2$, where $t_{\text {relay }}^{\text {En.D. }}$ is the time-gain of the attack against an energy detector.

\section{Vi. Performance Evaluation}

We evaluate the effectiveness of the distance-decreasing attacks with packet-based system simulations. We simulate a full IEEE 802.15.4a system including all the operations necessary to receive a packet: timing acquisition, estimation of the channel energy-delay profile, SFD detection, and data decoding. The physical layer is simulated with an accuracy of $100 \mathrm{ps}$. We use the residential non-line-of-sight channel model [29] with a channel delay spread $T_{\text {spread }} \approx 60 \mathrm{~ns}$. The signal 
TABLE I

Upper-bound on (relay) time-gain and (relay) distance-decrease of various PHY attacks in various "adversarial receiver against honest receiver" configurations. The left column presents conservative attacks, that work with $100 \%$ success probability. The right column presents the maximal additional time-gain/distance-decrease that can be achieved by combining PHY attacks and guessing attacks (when time guessing probability approaches the guessing probability of pure guessing attacks). Time-gain is expressed in terms of $T_{\mathrm{sym}}-$ payload symbol duration, $t_{\mathrm{ED}}=48-60 n s-$ detection time of honest receivers without ED-countermeasure, $t_{\mathrm{det}}^{\mathrm{A}}-$ detection time of the adversary, $t_{\mathrm{PLC}}<t_{\mathrm{det}}-$ pulse LC delay, $t_{\mathrm{THS}}^{\max }-$ maximum time-hopping offset, $t_{\mathrm{det}}^{C}=48-60 \mathrm{~ns}$ - detection time of honest receiver with ED-countermeasure, $t_{\mathrm{PLC}}^{C}<t_{\mathrm{det}}^{C}$ - pulse LC delay if countermeasure is deployed. The corresponding distance-decrease is shown for the IEEE 802.15.4a mandatory modes and delay values that maximize the distance-decrease.

\begin{tabular}{|c|c|c|c|c|c|}
\hline & & \multicolumn{2}{|l|}{ No guessing } & \multicolumn{2}{|c|}{ Max. guessing gain } \\
\hline & & (relay) time-gain & distance-decrease & (relay) time-gain & distance-decrease \\
\hline \multicolumn{6}{|c|}{ En.D. against En.D. } \\
\hline \multirow[t]{3}{*}{ Malicious Prover } & ED-only & $T_{\mathrm{sym}} / 4+\left(t_{\mathrm{det}}-t_{\mathrm{det}}^{\mathrm{A}}\right) / 2$ & $86 \mathrm{~m}$ & $+t_{\mathrm{THS}}^{\max }$ & $+74 \mathrm{~m}$ \\
\hline & LC-only & $T_{\mathrm{sym}} / 4+t_{\mathrm{PLC}} / 2$ & $86 \mathrm{~m}$ & $+t_{\mathrm{THS}}^{\max }$ & $+74 \mathrm{~m}$ \\
\hline & $\mathrm{ED}+\mathrm{LC}$ & $T_{\mathrm{sym}} / 2+\left(t_{\mathrm{PLC}}+t_{\mathrm{det}}-t_{\mathrm{det}}^{\mathrm{A}}\right) / 2$ & $171 \mathrm{~m}$ & $+t_{\mathrm{THS}}^{\max }$ & $+74 \mathrm{~m}$ \\
\hline Relay Attack & $\mathrm{ED}+\mathrm{LC}$ & $T_{\mathrm{sym}} / 2+t_{\mathrm{PLC}}-t_{\mathrm{det}}^{\mathrm{A}}$ & $171 \mathrm{~m}$ & +0 & $+0 \mathrm{~m}$ \\
\hline \multicolumn{6}{|c|}{ Rake against En.D. } \\
\hline \multirow[t]{2}{*}{ Malicious Prover } & ED-only & $T_{\mathrm{sym}} / 2+\left(t_{\mathrm{det}}-t_{\mathrm{det}}^{\mathrm{A}}\right) / 2$ & $162 \mathrm{~m}$ & $+T_{\mathrm{sym}} / 4+t_{\mathrm{THS}}^{\max }$ & $+151 \mathrm{~m}$ \\
\hline & $\mathrm{ED}+\mathrm{LC}$ & $3 / 4 \cdot T_{\mathrm{sym}}+\left(t_{\mathrm{PLC}}+t_{\mathrm{det}}-t_{\mathrm{det}}^{\mathrm{A}}\right) / 2$ & $248 \mathrm{~m}$ & $+T_{\mathrm{sym}} / 4+t_{\mathrm{THS}}^{\max }$ & $+151 \mathrm{~m}$ \\
\hline \multirow[t]{2}{*}{ Relay Attack } & $\mathrm{ED}+\mathrm{LC}$ & $T_{\mathrm{sym}}-t_{\mathrm{THS}}^{\max }+t_{\mathrm{PLC}}-t_{\mathrm{det}}^{\mathrm{A}}$ & $251 \mathrm{~m}$ & $+T_{\mathrm{sym}} / 2+2 \cdot t_{\mathrm{THS}}^{\mathrm{max}}$ & $+302 \mathrm{~m}$ \\
\hline & ED-only & $T_{\mathrm{sym}} / 2-t_{\mathrm{THS}}^{\mathrm{max}}-t_{\mathrm{det}}^{\mathrm{A}}$ & $79 \mathrm{~m}$ & $+T_{\mathrm{sym}} / 2+2 \cdot t_{\mathrm{THS}}^{\max }$ & $+302 \mathrm{~m}$ \\
\hline \multicolumn{6}{|c|}{ Rake against Rake } \\
\hline \multirow[t]{3}{*}{ Malicious Prover } & ED-only & $\left(t_{\mathrm{det}}-t_{\mathrm{det}}^{\mathrm{A}}\right) / 2$ & $5 \mathrm{~m}$ & $+T_{\mathrm{sym}} / 4+t_{\mathrm{THS}}^{\max }$ & $+151 \mathrm{~m}$ \\
\hline & LC-only & $t_{\mathrm{PLC}} / 2$ & $5 \mathrm{~m}$ & $+T_{\mathrm{sym}} / 4+t_{\mathrm{THS}}^{\max }$ & $+151 \mathrm{~m}$ \\
\hline & $\mathrm{ED}+\mathrm{LC}$ & $\left(t_{\mathrm{det}}+t_{\mathrm{PLC}}-t_{\mathrm{det}}^{\mathrm{A}}\right) / 2$ & $10 \mathrm{~m}$ & $+T_{\mathrm{sym}} / 2+t_{\mathrm{THS}}^{\max }$ & $+228 \mathrm{~m}$ \\
\hline Relay Attack & $\mathrm{ED}+\mathrm{LC}$ & $t_{\mathrm{PLC}}-t_{\mathrm{det}}^{\mathrm{A}}$ & $10 \mathrm{~m}$ & +0 & $+0 \mathrm{~m}$ \\
\hline \multicolumn{6}{|c|}{ En.D./Rake against En.D./Rake with ED-countermeasure (Section VII-3) and convolutional code patch } \\
\hline \multirow[t]{3}{*}{ Malicious Prover } & ED-only & $\left(t_{\mathrm{det}}^{\mathrm{C}}-t_{\mathrm{det}}^{\mathrm{A}}\right) / 2$ & $5-6 \mathrm{~m}$ & $+t_{\mathrm{THS}}^{\max }$ & $+74 \mathrm{~m}$ \\
\hline & LC-only & $t_{\mathrm{PLC}}^{C} / 2$ & $5-6 \mathrm{~m}$ & $+t_{\mathrm{THS}}^{\max }$ & $+74 \mathrm{~m}$ \\
\hline & $\mathrm{ED}+\mathrm{LC}$ & $\left(t_{\mathrm{PLC}}^{C}+t_{\mathrm{det}}^{\mathrm{C}}-t_{\mathrm{det}}^{\mathrm{A}}\right) / 2$ & $10-12 \mathrm{~m}$ & $+t_{\mathrm{THS}}^{\max }$ & $+74 \mathrm{~m}$ \\
\hline Relay Attack & $\mathrm{ED}+\mathrm{LC}$ & $t_{\mathrm{PLC}}-t_{\mathrm{det}}^{\mathrm{A}}$ & $10-12 \mathrm{~m}$ & +0 & $+0 \mathrm{~m}$ \\
\hline \multicolumn{6}{|c|}{ En.D./Rake against En.D./Rake with ED-countermeasure and convolutional code and time-hopping patches } \\
\hline \multirow[t]{3}{*}{ Malicious Prover } & ED-only & $\left(t_{\operatorname{det}}^{\mathrm{C}}-t_{\mathrm{det}}^{\mathrm{A}}\right) / 2$ & $5-6 \mathrm{~m}$ & +0 & $+0 \mathrm{~m}$ \\
\hline & LC-only & $t_{\mathrm{PLC}}^{C} / 2$ & $5-6 \mathrm{~m}$ & +0 & $+0 \mathrm{~m}$ \\
\hline & $\mathrm{ED}+\mathrm{LC}$ & $\left(t_{\mathrm{PLC}}^{C}+t_{\mathrm{det}}^{\mathrm{C}}-t_{\mathrm{det}}^{\mathrm{A}}\right) / 2$ & $10-12 \mathrm{~m}$ & +0 & $+0 \mathrm{~m}$ \\
\hline Relay Attack & $\mathrm{ED}+\mathrm{LC}$ & $t_{\mathrm{PLC}}-t_{\mathrm{det}}^{\mathrm{A}}$ & $10-12 \mathrm{~m}$ & +0 & $+0 \mathrm{~m}$ \\
\hline
\end{tabular}

to noise ratio (SNR) is defined as $\mathrm{SNR}=\frac{E_{p}}{N_{0}}$ where $E_{p}$ is the received energy per pulse (after the convolution of the pulse with the impulse response of the channel). We assume a desired security level of $P_{\text {guess }}=2^{-32}$, and performance goals $\mathrm{PER}_{\mathrm{comm}}=\mathrm{PER}_{\mathrm{db}}=10^{-2}$. According to Section III-B, this results in ranging packets of length $N_{\text {nonce }}=42$ with a maximum of $N_{\text {err }}=2$ tolerable bit errors.

\section{A. Energy Detector against Energy Detector}

For the energy detector against energy detector setting, a detailed performance evaluation of the ED and LC components, as well as of their combination in the case of the relay attack, was already presented in our previous paper [4] as well as in [32]. For a detailed discussion of the results, as well as alternative energy-detection receivers (which show similar performance), we therefore refer the reader there. In the following we give a summary of our main findings:

- In all attack scenarios, an adversary can decrease the distance by an amount that is close to the upper-bounds given in Table I. Furthermore, it can do so with an impressive success rate of $99 \%$ and at a cost of just a few dB in SNR with respect to normal system operation. A further increase in
SNR allows the adversary to make the success rate arbitrarily large.

- For example, to mount an ED attack on the payload, achieving a distance-decrease of $77 \mathrm{~m}\left(t_{\mathrm{det}}=t_{\mathrm{det}}^{\mathrm{A}}\right.$ in the order of the channel delay spread) with a success rate of $99 \%$, the adversary requires an SNR at ARX that is $1.6 \mathrm{~dB}$ higher than in benign-case operation. A LC attack on the payload achieving the same distance-decrease (i.e., $t_{\mathrm{PLC}}=0$ ) costs $4 \mathrm{~dB}$ in SNR.

- A relay attack, including attacks on both preamble and payload, and achieving a distance-decrease of $144 \mathrm{~m}\left(t_{\mathrm{PLC}}=\right.$ $\left.0, t_{\mathrm{det}}^{\mathrm{A}}=32 \mathrm{~ns}\right)$, requires $6 \mathrm{~dB}$ higher SNR at $\mathrm{ARX}$ and $4 \mathrm{~dB}$ higher SNR at HRX, compared to benign-case operation.

\section{B. Rake against Energy Detector}

We evaluate the rake ED attack for an optimal all-rake receiver with perfect synchronization and channel estimation. Figure 7 shows the PER for an adversary performing the ED attack. When mounting the ED attack, ARX has the option to ignore ("No Coding") or take advantage of the convolutional code ("Coding"). For reference, the performance of a benign energy-detection receiver and a benign rake receiver are shown 


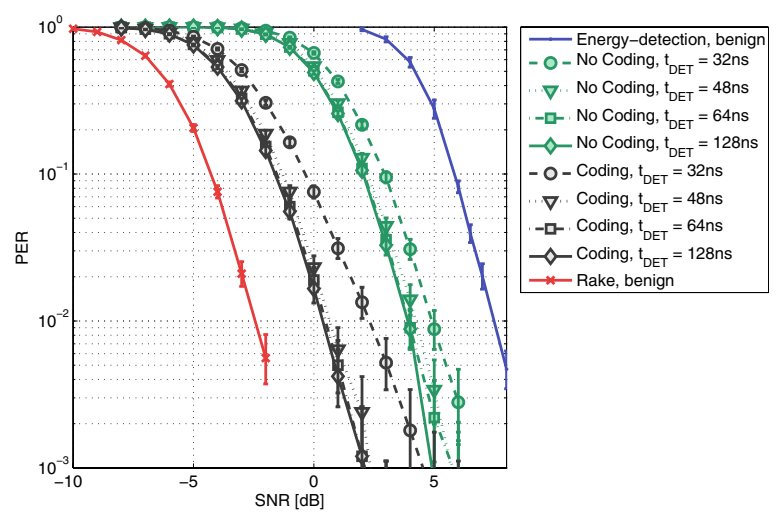

Fig. 7. Performance of early detection attack on the payload if the adversary uses an rake receiver. Early detection can be performed by decoding the convolutional code using partial information ("Coding") or by neglecting the convolutional code completely ("No Coding").

as well. The benign rake receiver decodes the convolutional code at the end of the packet as in [31], when the full decoding trellis is available. With ED and taking the code into account, only a partial trellis containing information about the symbols received so far is available at the time of decoding. This contributes to the higher cost (in terms of required SNR) of the attack with respect to the benign rake receiver operation. Ignoring the convolutional code is simpler and less computationally expensive, but results in an additional $3.5 \mathrm{~dB}$ increase of the attack cost.

The adversary also has the choice of $t_{\mathrm{det}}^{\mathrm{A}}$. Optimal performance is experienced for $t_{\mathrm{det}}^{\mathrm{A}}=64 \mathrm{~ns}$, in the order of channel spread. $t_{\mathrm{det}}^{\mathrm{A}}=48 \mathrm{~ns}$ results in a very minor performance loss, $t_{\mathrm{det}}^{\mathrm{A}}=32 \mathrm{~ns}$ results in noticeable performance loss (around $1-2 \mathrm{~dB}$ ). Assuming $t_{\mathrm{det}}^{\mathrm{A}}=48 \mathrm{~ns}$, the attack costs $2.8 \mathrm{~dB}$ (at a PER of $10^{-2}$, corresponding to an attack with a success rate of $99 \%$ ) if coding is taken into account and the relay attack achieves a time-gain of $t_{\text {relay }}=728 \mathrm{~ns}$ (distance-decrease of 218 meters). At the same cost, the alternative, ED-only attack achieves a time-gain of $t_{\text {relay }}^{\mathrm{ED}-\text { anly }}=216 \mathrm{~ns}$ (distance-decrease of 65 meters).

\section{Countermeasures}

When investigating countermeasures and patches, we consider their effectiveness (the maximum relay time-gain the adversary can achieve with the countermeasure in place), the effects they have on benign-case performance, and their compatibility with IEEE 802.15.4a. We discuss here the countermeasures which, in our opinion, provide the best trade-off between these factors.

1) Convolutional Code Patch: The rake ED attack is possible due to the specific combination of BPPM/BPSK with the convolutional code that IEEE 802.15.4a uses. The attack can be avoided with a different convolutional code, for which the polarity bit $a_{i}$ does not reveal information about future position bits $d_{j}$, where $j>i$. The code should not allow for decoding $a_{i+1}$ from the $i$-th symbol $i$, as this would enable an effective LC attack against a rake receiver. We refer to this solution as the convolutional code patch. Alternatively, the convolutional code can be removed altogether. The

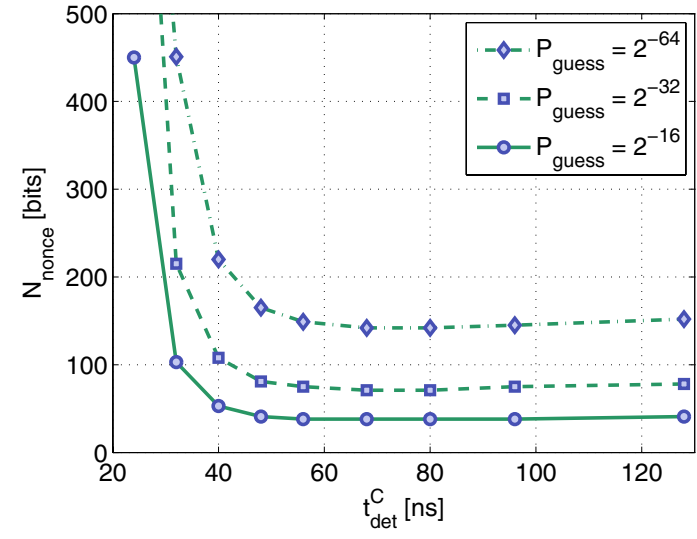

Fig. 8. Cost of Section VII-3 countermeasure when the energy-detection receiver decides on the bit value using OOK demodulation at time $t_{\mathrm{det}}^{\mathrm{C}}$.

former is not compatible with IEEE 802.15.4a, whereas the latter is compatible to a very limited extent: Although IEEE 802.15.4a provides a few optional modes that do not use the convolutional code, these modes cannot be used with energydetection receivers, because, for these modes, polarity carries data information.

2) Time-Hopping Patch: Time-hopping allows a malicious prover to trade-off attack success probability for additional time-gain. A simple way to remove this vulnerability is to modify the time-hopping sequence such that the corresponding challenge and response symbols have identical time-hopping offsets. Removing time-hopping is also a solution, but an inferior one, as it significantly degrades IEEE 802.15.4a multiuser access properties.

3) Early Detection at Honest Receiver: The honest energydetection receiver can choose to only take into account the beginning of the symbol [16], essentially performing early detection with OOK demodulation at an offset $t_{\mathrm{det}}^{\mathrm{C}}$ from the beginning of the symbol. Then, $t_{\mathrm{LC}}$ is reduced from $T_{\text {sym }} / 2$ to $t_{\mathrm{PLC}}^{C}<t_{\mathrm{det}}^{\mathrm{C}}$, and the (malicious prover) time-gain due to ED is limited to $\left(t_{\mathrm{det}}^{\mathrm{C}}-t_{\mathrm{det}}^{\mathrm{A}}\right) / 2$ (assuming that the Prover sends the response symbol immediately after the early detection is done). This countermeasure does not induce inter-symbol interference and is compliant with the mandatory modes of the standard.

Moreover, the mild performance loss that this countermeasure entails due to the ignoring of half of the symbol, can be compensated for by increasing the length of the nonces $N_{\text {nonce }}$. We can derive the required nonce length by using the method introduced in Section III-B. Figure 8 plots the resultant $N_{\text {nonce }}$ as a function of $t_{\text {det }}^{\mathrm{C}}$ for performance goals $\mathrm{PER}_{\mathrm{comm}}=\mathrm{PER}_{\mathrm{db}}=10^{-2}$ and 3 security levels. For example, by employing the countermeasure with $t_{\mathrm{det}}^{\mathrm{C}}=40 \mathrm{~ns}$ and increasing the number of bits per nonce from 42 to 108 we can bring the maximum theoretically achievable time-gain to $40 \mathrm{~ns}$ (distance decrease of about $12 \mathrm{~m}$ ) maintaining security level $P_{\text {guess }}=2^{-32}$. At the same time, this countermeasure does not reduce the performance in terms of PER and we also keep the same security level against guessing attacks. The only drawback is generating, sending and receiving of the additional bits required for the longer nonces. As every 
IEEE 802.15 .4 a payload carrying a nonce is preceded by a preamble of considerable length, and as a good deal of receiver complexity during reception stems from synchronization, we argue that the cost of adding a few bits to the payload is in most cases acceptable.

Furthermore, this countermeasure can be employed by both energy-detection and rake receivers to prevent the adversary from exploiting the BPPM variability (Section V-B). See Table I for upper-bounds on the attack time-gains with the countermeasure and patches deployed.

\section{CONCLUSION}

We have investigated the vulnerability of the IEEE 802.15.4a standard to physical layer distance-decreasing attacks. We have demonstrated that if honest devices use energydetection receivers without appropriate countermeasures, an adversary can decrease the measured distance by hundreds of meters, with a success rate arbitrarily close to $100 \%$. However, minor modifications to IEEE 802.15.4a and implementing a simple countermeasure on energy-detection receivers used by honest devices, allow honest devices to reduce the effectiveness of distance-decreasing relay attacks to at most $10 \mathrm{~m}$. Alternatively, this can be achieved (even without IEEE 802.15.4a modifications) if honest devices use the more sophisticated rake receivers. Furthermore, to reduce the effectiveness of malicious prover attacks to around $10 \mathrm{~m}$, the honest receivers (energy detector and rake alike) should implement the same simple countermeasure, and a time-hopping patch should be applied to IEEE 802.15.4a. In conclusion, with appropriate countermeasures on the receivers and patches to the IEEE 802.15.4a standard, the standard can be used as a DB PHY.

More generally, our investigation has identified PHY features that, although improving system performance in the benign case, can create vulnerabilities against distancedecreasing PHY attacks if used carelessly. One such potential point of failure is the interaction between the modulation and the coding scheme. Another, perhaps more fundamental one, is payload time-hopping, which allows the adversary to additionally decrease the distance by lowering the attack's probability of success. Such features should be approached with caution, or not used at all, in any DB PHY.

\section{REFERENCES}

[1] S. Gezici, Z. Tian, G. Giannakis, H. Kobayashi, A. Molisch, H. V. Poor, and Z. Sahinoglu, "Localization via ultra-wideband radios: a look at positioning aspects for future sensor networks," IEEE Signal Process. Mag., vol. 22, no. 4, July 2005.

[2] S. Brands and D. Chaum, "Distance-bounding protocols," in EUROCRYPT'93, Lect. Notes in Computer Science 765, 1993.

[3] J. Clulow, G. P. Hancke, M. G. Kuhn, and T. Moore, "So near and yet so far: distance-bounding attacks in wireless networks," in Third European Workshop on Security and Privacy in Ad-hoc and Sensor Networks, 2006. Available: http://www.crysys.hu/ESAS2006/cfp.html

[4] M. Flury, M. Poturalski, P. Papadimitratos, J.-P. Hubaux, and J.Y. Le Boudec, "Effectiveness of distance-decreasing attacks against impulse radio ranging," in $3 r d$ ACM Conference on Wireless Network Security, 2010.

[5] M. Poturalski, M. Flury, P. P, J.-P. Hubaux, and J.-Y. Le Boudec, "Distance bounding with ieee 802.15.4a: attacks and countermeasures," Tech. Rep. EPFL-REPORT-150112, Jan. 2011.

[6] G. Hancke and M. Kuhn, "An RFID distance bounding protocol," in First International Conference on Security and Privacy for Emerging Areas in Communications Networks, 2005.
[7] S. Čapkun and J.-P. Hubaux, "Secure positioning in wireless networks," IEEE J. Sel. Areas Commun., vol. 24, no. 2, 2006.

[8] D. Singelée and B. Preneel, "Distance bounding in noisy environments," in European Workshop on Security and Privacy in Ad-Hoc and Sensor Networks, 2007.

[9] L. Bussard, "Trust establishment protocols for communicating devices," Ph.D. dissertation, 2004.

[10] J. Reid, J. M. G. Nieto, T. Tang, and B. Senadji, "Detecting relay attacks with timing-based protocols," in ASIAN ACM Symposium on Information, Computer and Communications Security, 2007.

[11] C. H. Kim, G. Avoine, F. Koeune, F.-X. Standaert, and O. Pereira, "The swiss-knife RFID distance bounding protocol," in International Conference on Information Security and Cryptology, P. Lee and J. Cheon, editors, 2008

[12] C. H. Kim and G. Avoine, "RFID distance bounding protocol with mixed challenges to prevent relay attacks," in 8th International Conference on Cryptology and Network Security, 2009. Available: http://www.uclouvain.be/sites/security/download/papers/KimA-2009cans.pdf.

[13] K. B. Rasmussen and S. Čapkun, "Location privacy of distance bounding protocols," in 15th ACM conference on Computer and Communications Security, 2008.

[14] C. Meadows, R. Poovendran, D. Pavlovic, L.-W. Chang, and P. Syverson, "Distance bounding protocols: authentication logic analysis and collusion attacks," in Secure Localization and Time Synchronization for Wireless Sensor and Ad Hoc Networks. Springer-Verlag, Series: Advances in Information Security, vol. 30, 2007.

[15] P. Schaller, B. Schmidt, D. Basin, and S. Čapkun, "Modeling and verifying physical properties of security protocols for wireless networks," in Proc. 22nd IEEE Computer Security Foundations Symposium, 2009.

[16] G. P. Hancke and M. G. Kuhn, "Attacks on time-of-flight distance bounding channels," in First ACM conference on Wireless Network Security, 2008.

[17] M. Poturalski, M. Flury, P. Papadimitratos, J.-P. Hubaux, and J.-Y. Le Boudec, "The cicada attack: degradation and denial of service in IR ranging," in IEEE International Conference on Ultra-Wideband, 2010.

[18] M. Kuhn, H. Luecken, and N. Tippenhauer, "UWB impulse radio based distance bounding," in Proc. Workshop on Positioning, Navigation and Communication, 2010.

[19] N. O. Tippenhauer and S. Čapkun, "ID-based secure distance bounding and localization," in Proc. European Symposium on Research in Computer Security, 2009.

[20] J. Munilla and A. Peinado, "Distance bounding protocols for RFID enhanced by using void-challenges and analysis in noisy channels," Wireless Commun. and Mobile Computing, vol. 8, no. 9, 2008.

[21] G. Hancke, "Design of a secure distance-bounding channel for RFID," Elsevier J. Network and Computer Applications, 2010.

[22] S. Drimer and S. Murdoch, "Keep your enemies close: distance bounding against smartcard relay attacks," in Proc. 16th USENIX Security Symposium, 2007.

[23] Y. Desmedt, "Major security problems with the "unforgeable"(Feige)Fiat-Shamir proofs of identity and how to overcome them," in SecuriCom, 1988.

[24] IEEE Computer Society, LAN/MAC Standard Committee, "IEEE P802.15.4a/D7 (amendment of IEEE std 802.15.4), part 15.4: Wireless medium access control (MAC) and physical layer (PHY) specifications for low-rate wireless personal area networks," Jan. 2007.

[25] C. Duan, P. Orlik, Z. Sahinoglu, and A. F. Molisch, "A non-coherent 802.15.4a UWB impulse radio," in IEEE International Conference on Ultra-Wideband, Sep. 2007, pp. 146-151.

[26] J. Ryckaert, G. Van der Plas, V. De Heyn, C. Desset, G. Vanwijnsberghe, B. Van Poucke, and J. Craninckx, "A 0.65-to-1.4nJ/burst 3-to$10 \mathrm{GHz}$ UWB digital TX in 90nm CMOS for IEEE 802.15.4a," IEEE International Solid-State Circuits Conference, 2007.

[27] A. A. D'Amico, U. Mengali, and E. Arias-De-Reyna, "Energy-detection UWB receivers with multiple energy measurements," IEEE Trans. Wireless Commun., vol. 6, no. 7, pp. 2652-2659, 2007.

[28] M. Flury, R. Merz, and J.-Y. Le Boudec, "An energy detection receiver robust to multi-user interference for ieee 802.15.4a networks," in IEEE International Conference on Ultra-Wideband, 2008.

[29] A.-F. Molisch, K. Balakrishnan, C.-C. Chong, S. Emami, A. Fort, J. Karedal, J. Kunisch, H. Schantz, U. Schuster, and K. Siwiak, "IEEE 802.15.4a channel model-final report, document 04/662r1," Nov. 2004.

[30] J. G. Proakis and M. Salehi, Digital Communications, 5th edition. McGraw-Hill, 2008.

[31] Z. Ahmadian and L. Lampe, "Performance analysis of the IEEE 802.15.4a UWB system," IEEE Trans. Commun., vol. 57, no. 5, pp. 1474-1485, 2009. 
[32] M. Flury, "Interference robustness and security of impulse-radio ultrawide band networks," Ph.D. dissertation, Lausanne, 2010. Available: http://library.epfl.ch/theses/?nr=4698

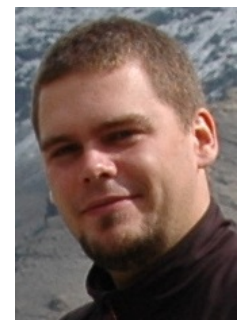

Marcin Poturalski earned his Master of Science degree in Computer Science and Bachelor Degree in Mathematics from Warsaw University in 2005 During his studies he completed internships $\mathrm{Mi}-$ crosoft Research Asia in Beijing and Microsoft Corp. in Redmond, USA. In 2006 he joined the Laboratory for Computer Communication and Applications (LCA) at EPFL, School of Computer and Communication Sciences. He is pursuing his $\mathrm{PhD}$ degree under the supervision of Jean-Pierre Hubaux and Panos Papadimitratos. He is participating in the National Center of Competence in Research project on Mobile Information and Communication Systems (NCCR-MICS). His research interests are in security of wireless communication, notably neighbor discovery, ranging and localization.

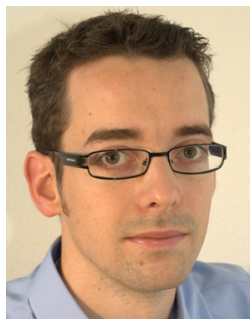

Manuel Flury earned his Master of Science degree in Communication Systems Engineering from Ecole Polytechnique Federale de Lausanne (EPFL) in 2005. During his studies he completed internships at Nokia Research Center in Helsinki, Finland and at Qualcomm Inc. in San Diego, USA. In 2005 he joined the Laboratory for Computer Communication and Applications (LCA) at EPFL, School of Computer and Communication Sciences, and began working on his Ph.D. thesis. There, he participated in the National Center of Competence in Research project on Mobile Information and Communication Systems (NCCR-MICS) and he earned his Ph.D. in 2010. His research interests are in wireless communication and computer networks.

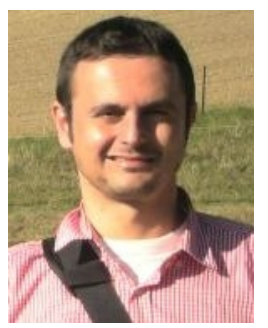

Panos Papadimitratos earned his $\mathrm{PhD}$ from Cornell University, Ithaca, NY, USA, in 2005. After being a post-doctoral fellow at Virginia Tech, Blacksburg, VA, USA, and a scientist at EPFL, Lausanne, Switzerland, he joined the faculty of KTH, Stockholm, Sweden, where he is now an Associate Professor in the School of Electrical Engineering. His research is concerned with security and networked systems, with more than 70 related technical publications. He has delivered a number of invited talks and tutorials, including tutorials at the ACM CCS and the ACM MobiCom conferences. He is an Area Editor for the ACM $\mathrm{MC} 2 \mathrm{R}$ journal and he has served in several technical program committees, including IEEE INFOCOM and ACM WiSec, ASIACCS, and MobiHoc. His webpage is: http://www.ee.kth.se/ papadim/

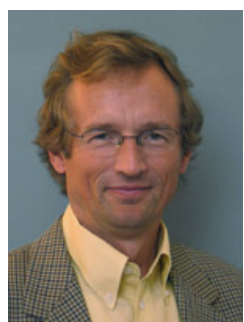

Jean-Pierre Hubaux joined the faculty of EPFL in 1990. He became full professor in 1996. He is a Fellow of both ACM and IEEE. His current research activity is focused on privacy preservation mechanisms in pervasive communications.

In 1991, he designed the first curriculum in Communication Systems at EPFL. He was promoted to full professor in 1996. In 1999, he defined some of the main ideas of the National Competence Center in Research named "Mobile Information and Communication Systems" (NCCR/MICS); this center (still very active) was initially nicknamed "the Terminodes Project". In this framework, he has notably defined, in close collaboration with his students, novel schemes for the security and cooperation in wireless networks; in particular, he has devised new techniques for key management, secure positioning, and incentives for cooperation in such networks. In 2003, he identified the security of vehicular networks as one of the main research challenges for real-world mobile ad hoc networks. In 2008, he completed a graduate textbook entitled Security and Cooperation in Wireless Networks, with Levente Buttyan. Some of his current research activities are funded by Nokia.

$\mathrm{He}$ is co-founder and chairman of the steering committee of WiSec (the ACM Conference for Wireless Network Security). He has served on the program committees of numerous conferences and workshops, including SIGCOMM, INFOCOM, MobiCom, MobiHoc, SenSys, WiSe, WiSec and VANET. He is one of the seven commissioners of the Federal Communications Commission (ComCom), the "Swiss FCC".

$\mathrm{He}$ was born in Belgium, but spent most of his childhood and youth in Northern Italy. After completing his studies in Electrical Engineering at Politecnico di Milano, he worked 10 years in France with Alcatel, primarily in the area of switching systems architecture and software. He held visiting positions at the IBM T.J. Watson Research Center and at UC Berkeley.

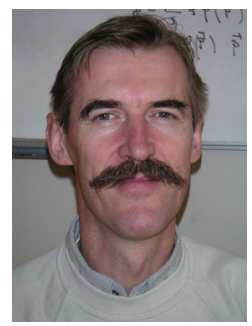

Jean-Yves Le Boudec is full professor at EPFL and fellow of the IEEE. He graduated from Ecole Normale Superieure de Saint-Cloud, Paris, where he obtained the Agregation in Mathematics in 1980 (rank 4) and earned his doctorate in 1984 from the University of Rennes, France. From 1984 to 1987 he was with INSA/IRISA, Rennes. In 1987 he joined Bell Northern Research, Ottawa, Canada, as a member of scientific staff in the Network and Product Traffic Design Department. In 1988, he joined the IBM Zurich Research Laboratory where he was manager of the Customer Premises Network Department. In 1994 he joined EPFL as associate professor.

His interests are in the performance and architecture of communication systems. In 1984, he developed analytical models of multiprocessor, multiple bus computers. In 1990 he invented the concept called "MAC emulation" which later became the ATM forum LAN emulation project, and developed the first ATM control point based on OSPF. He also launched public domain software for the interworking of ATM and TCP/IP under Linux. He proposed in 1998 the first solution to the failure propagation that arises from common infrastructures in the Internet. He contributed to network calculus, a recent set of developments that forms a foundation to many traffic control concepts in the internet, and co-authored a book on this topic. He is also the author of the book Performance Evaluation (2010). He received the IEEE Millenium Medal, the Infocom 2005 Best Paper award, the CommSoc 2008 William R. Bennett Prize and the 2009 ACM Sigmetrics Best Paper award.

$\mathrm{He}$ is or has been on the program committee or editorial board of many conferences and journals, including Sigcomm, Sigmetrics, Infocom, Performance Evaluation and ACM/IEEE TRANSACTIONS ON NETWORKING. 